\title{
Evolution of Structure Functions And Their Moments In Chiral Field Theory
}

\author{
Kakali Ray-Maity \\ and \\ Padmanabha Dasgupta \\ Department of Physics, University of Kalyani \\ West Bengal, India 741235
}

\begin{abstract}
Evolution of structure functions and their moments at low and moderate $Q^{2}$ is studied in the chiral field theory. Evolution equations based on perturbation expansion in the coupling constant of the effective theory are derived and solved for the moments. The kernels of evolution arising from different processes have been calculated with contributions from direct and cross channels, the interference terms being non-negligible in the kinematic regions under consideration. This is shown to lead to flavor-dependence of the kernels which manifests in observable effects. The invalidity of the probabilistic approach to the evolution process is also pointed out.
\end{abstract}




\section{Introduction}

While perturbative QCD has been reasonably successful in describing the scale dependence of the structure functions at asymptotically large $Q^{2}$ [1], phenomena observed experimentally at intermediate $Q^{2}$ seem to demand consideration of nonperturbative effects[2], the observed departure of the Gottfried sum from the parton model value providing a notable example [3]. Effective theories based on $q q \pi$ vertex, which are expected to incorporate the relevant nonperturbative effects, give simple, and at least qualitative, explanation of the observed phenomena[4-6].

In such theories, the QCD evolution of the effective parton densities due to quarkgluon interaction is replaced essentially by evolution due to quark pion interaction. The evolution equations proposed in these theories involve evolution kernels or splitting functions which can be computed from the virtual photon cross sections for pion emission processes. While in perturbative QCD, the evolution kernels result directly from the Weiszäcker-Williams procedure and admit simple probabilistic interpretation, we point out in this paper that the correct evolution kernels in pion-emission theories are not consistent with such interpretation.

Although we illustrate the point with the specific example of the chiral field theory [7] used in [4], the conclusions are absolutely general.

The effective quark densities are defined in the Altarelli -Parisi formalism through the use of leading log approximation. In effective theories, the lack of asymptotoic freedom requires a different procedure for defining the effective quark densities. We discuss this in the next section. In section 3, the evolution equations for the effective parton densities thus defined are obtained. In section 4, we also show the evolution equations that would follow from the probabilistic approach in a kinematic region where such approach may be supposed to be valid and point out the difficulties that would result from such a procedure. In section 5, we discuss the evolution of the moments of the structure function that follow from the evolution equations derived in section 3.

\section{Effective quark densities}

The standard theoretical approach to the $Q^{2}$-dependence of the structure functions begins with the formula

$$
F_{2}^{e N}\left(x, Q^{2}\right) / x=\sum_{i} e_{i}^{2} \int \frac{d y}{y} q_{i \circ}(y) \sigma^{\gamma^{*} q_{i}}\left(x / y, Q^{2}\right)
$$

where $q_{i \circ}$ is the naive parton model density of the $i$-th parton in the target nucleon and $\sigma^{\gamma^{*}} q_{i}$ is the $\gamma^{*} q_{i}$ cross section integrated over $p_{T}^{2}$ and made adimensional by 
taking out a scale factor [1]. Correction to the naive parton model results comes entirely from the cross section which in QCD and in effective theories is quite different from the $Q^{2}$-independent point-like cross section of the former.

To analyze the scale dependence of the structure functions from (2.1), a convenient formalism is obtained by introducing the effective parton densities $q_{i}\left(x, Q^{2}\right)$ and setting up evolution equations for them. It is well known that in perturbative QCD this is done in the leading logarithm approximation by resumming the ladder diagrams in the perturbation series, factoring it out and absorbing it in the definition of the parton densities. In the case of the effective theories we are concerned with, it must be remembered however that (i) these theories are not asymptotically free, and (ii) the region of interest to which one would like to use these is not the asymptotic but the moderate $Q^{2}$ region.

All one can do here is expand the cross section in terms of the effective quark-pion coupling constant $\alpha_{\pi}$.

$$
\sigma\left(z, Q^{2}\right)=\delta(z-1)+\frac{\alpha_{\pi}}{2 \pi} \sigma_{\alpha}\left(z, Q^{2}\right)+O\left(\alpha_{\pi}^{2}\right)
$$

and redefine the parton densities as

$$
q_{i}\left(x, Q^{2}\right)=q_{i \circ}(x)+\Delta q_{i}\left(x, Q^{2}\right)
$$

with

$$
\Delta q_{i}\left(x, Q^{2}\right)=\frac{\alpha_{\pi}}{2 \pi} \sum_{j} \int_{x}^{1} \frac{d y}{y} q_{j \circ}(y) \sigma_{\alpha}^{i j}\left(x / y, Q^{2}\right)+O\left(\alpha_{\pi}^{2}\right)
$$

so that one recovers

$$
F_{2}^{e N}\left(x, Q^{2}\right) / x=\sum_{i} e_{i}^{2} q_{i}\left(x, Q^{2}\right)
$$

which is the key formula in any theory of this kind. In $(2.4), \sigma_{\alpha}^{i j}$ denotes the lowest order cross section for the process $\gamma^{*} q_{j} \rightarrow q_{i} \pi$.

Then with the standard notation $\tau=\log \left(Q^{2} / \mu^{2}\right), \mu^{2}$ being a parameter defining the $Q^{2}$ scale one can write down the evolution equation to the lowest order in $\alpha_{\pi}$

$$
\frac{d}{d \tau} q_{i}(x, \tau)=\sum_{j} \mathcal{P}_{i j} \otimes q_{j}
$$

where

$$
\begin{gathered}
\mathcal{P}_{i j} \otimes q_{j}=\int \frac{d y}{y} \mathcal{P}_{i j}(x / y, \tau) q_{j}(y, \tau) \\
\mathcal{P}_{i j}(z, \tau)=\frac{d}{d \tau} \sigma_{\alpha}^{i j}(z, \tau)
\end{gathered}
$$


The last equation does not by itself guarantee universality or process-independence of the evolution kernels, an elegant feature of the splitting functions appearing in the leading log QCD evolution.

The crucial point is that emission or absorption of an isovector causes a change of flavor of the initial quark. The lowest order cross section for the process $\gamma^{*} q \rightarrow q^{\prime} \pi$ can be written as (after taking out the scale factor)

$$
\sigma_{\alpha}^{q \prime q}\left(x, Q^{2}\right)=\sigma_{d}^{q \prime q}\left(x, Q^{2}\right)+\frac{e_{q \prime}}{e_{q}} \sigma_{i}^{q \prime q}\left(x, Q^{2}\right)
$$

The cross-section for the process $\gamma^{*} \pi \rightarrow q^{\prime} \bar{q}$ can be written as

$$
\sigma_{\alpha}^{q^{\prime} \pi}=\sigma_{d}^{q^{\prime} \pi}\left(x, Q^{2}\right)+\left(\frac{e_{\pi}}{e_{q}^{\prime}}-1\right) \sigma_{i}^{q^{\prime} \pi}\left(x, Q^{2}\right)
$$

where $\sigma_{d}$ is the direct ( $t$-channel exchange) cross section and $\sigma_{i}$ arises from the interference with the crossed channel ( $s$-channel exchange) diagram. Both the terms are of the same order in $\alpha_{\pi}$ and none of them can be neglected in the kinematic region of moderate $Q^{2}$ and moderate energy to which the theory is intended to be applied.

On the other hand, the cross section for the process $\gamma^{*} q \rightarrow \pi q^{\prime}$ can be written as

$$
\sigma_{\alpha}^{\pi q}\left(x, Q^{2}\right)=\sigma_{d}^{\pi q}\left(x, Q^{2}\right)
$$

This together with (2.8) leads to evolution kernels such that

$$
\mathcal{P}_{q^{\prime} q}\left(1-x, Q^{2}\right) \neq \mathcal{P}_{\pi q}\left(x, Q^{2}\right)
$$

This makes it impossible to sustain the probabilistic meaning of the evolution kernels. Further, the probabilistic interpretation would contradict the flavor-dependence of the evolution kernels $\mathcal{P}_{u \pi^{+}} \neq \mathcal{P}_{d \pi^{-}}$as that would apparently violate the $\mathrm{SU}(2)$ symmetry. While this adds to the flavor-asymmetry of the sea distribution [9] which in any case would arise in such a theory due to a multiplicatively evolving part of the Gottfried sum [2] and may help explain the observed asymmetry, the probabilistic interpretation of the evolutions is clearly traded off.

\section{Evolution equation in chiral field theory}

For a specific case, we focus our attention to the chiral field theory [7] which was invoked by Eichten et al.[4] in their explanation of the NMC result on the Gottfried sum rule. The linearized effective lagrangian is

$$
\mathcal{L}_{\Pi q}=-\frac{g_{A}}{f} \bar{\psi} \partial_{\mu} \Pi \gamma^{\mu} \gamma^{5} \psi
$$


Here $\Pi$ is the pseudoscalar octet of flavor $\mathrm{SU}(3)$ playing the role of Goldstone bosons, $\psi$ the quark field, $g_{A}$ the dimensionless axial vector coupling constant and $f$ the pseudoscalar decay constant.

Contributions from direct cross sections

1. $\sigma_{d}\left(\gamma^{*} q \rightarrow \pi q^{\prime}\right)$

The cross section for the process $\gamma^{*} q \rightarrow \pi q^{\prime}$ arising from the $\operatorname{direct}(\mathrm{t}$ channel) term is found to be of the form

$$
\sigma_{d}\left(\gamma^{*} q \rightarrow \pi q^{\prime}\right)=\frac{g_{A}^{2}}{f^{2}} \frac{\sigma_{\circ}}{64 \pi^{2}} \frac{1}{P^{2}} 2 m_{\pi}^{2}\left(m_{q}+m_{q^{\prime}}\right)^{2} \int_{-\Lambda^{2}}^{t_{2}} d t \frac{\left(m_{q}-m_{q}^{\prime}\right)^{2}-t}{\left(t-m_{\pi}^{2}\right)^{2}}
$$

where

$$
\begin{aligned}
\Lambda^{2} & =\min \left[-2 m_{q}^{2}+2 \sqrt{\left(P^{2}+m_{q}^{2}\right)\left(K^{2}+m_{q}^{2}\right)}+2 P K, \Lambda_{\chi S B}^{2}\right] \\
t_{2} & =2 m_{q}^{2}-2 \sqrt{\left(P^{2}+m_{q}^{2}\right)\left(K^{2}+m_{q}^{2}\right)}+2 P K \\
P^{2} & =\frac{\left(s+Q^{2}+m_{q}^{2}\right)^{2}}{4 s}-m_{q}^{2} \\
K^{2} & =\frac{\left(s-m_{q}^{2}-m_{\pi}^{2}\right)^{2}-4 m_{q}^{2} m_{\pi}^{2}}{4 s}
\end{aligned}
$$

and $\Lambda_{\chi S B}^{2}$ is the cut-off parameter of the chiral field theory and corresponds to the scale of chiral symmetry breaking. The flavor factor has been suppressed in this and all the formulae given below.

2. $\sigma_{d}\left(\gamma^{*} q_{i} \rightarrow q_{j} \pi\right)$

The cross section for the process $\gamma^{*} q_{i} \rightarrow q_{j} \pi$ arising from the direct (t channel) term is found to be of the from

$$
\sigma_{d}\left(\gamma^{*} q_{i} \rightarrow q_{j} \pi\right)=\frac{g_{A}^{2}}{f^{2}} \frac{\sigma_{\circ}}{64 \pi^{2}} \frac{1}{P^{2}} \int_{-\Lambda^{2}}^{t_{2}} d t \frac{a_{1}+b_{1} t+c_{1} t^{2}+d_{1} t^{3}}{\left(t-m_{q}^{2}\right)^{2}}
$$

where

$$
\begin{aligned}
\Lambda^{2} & =\min \left[2 \sqrt{\left(P^{2}+m_{q}^{2}\right)\left(K^{2}+m_{\pi}^{2}\right)}+2 P K-\left(m_{q}^{2}+m_{\pi}^{2}\right), \Lambda_{\chi S B}\right] \\
t_{2} & =-2 \sqrt{\left(P^{2}+m_{q}^{2}\right)\left(K^{2}+m_{\pi}^{2}\right)}+2 P K+m_{q}^{2}+m_{\pi}^{2} \\
K^{2} & =\frac{\left(s-m_{q}^{2}-m_{\pi}^{2}\right)^{2}-4 m_{q}^{2} m_{\pi}^{2}}{4 s} \\
P^{2} & =\frac{\left(s+Q^{2}-m_{q}^{2}\right)^{2}+4 m_{q}^{2} Q^{2}}{4 s}
\end{aligned}
$$


The expressions for the coefficients $a_{1}, b_{1}, c_{1}$ and $d_{1}$ in the integrand are given in the appendix.

3. $\gamma^{*} \pi \rightarrow q_{i} \bar{q}_{j}$

The cross section for the process $\gamma^{*} \pi \rightarrow q_{i} \bar{q}_{j}$ arising from the direct (t channel) term is found to be of the from

$$
\sigma_{d}\left(\gamma^{*} \pi \rightarrow q_{i} \bar{q}_{j}\right)=\frac{g_{A}^{2}}{f^{2}} \frac{\sigma_{\circ}}{32 \pi^{2}} \frac{1}{K^{2}} \int_{-\Lambda^{2}}^{t_{2}} d t \frac{a_{2}+b_{2} t+c_{2} t^{2}}{\left(t-m_{q}^{2}\right)^{2}}
$$

where

$$
\begin{aligned}
\Lambda^{2} & =\min \left[\frac{1}{2}\left(s+Q^{2}-m_{\pi}^{2}\right)+2 P K-m_{q}^{2}, \Lambda_{\chi S B}^{2}\right] \\
t_{2} & =-\frac{1}{2}\left(s+Q^{2}-m_{\pi}^{2}\right)+2 P K+m_{q}^{2} \\
K^{2} & =\frac{\left(s+Q^{2}+m_{\pi}^{2}\right)^{2}}{4 s}-m_{\pi}^{2} \\
P^{2} & =\frac{s-4 m_{q}^{2}}{4}
\end{aligned}
$$

The expressions for the $a_{2}, b_{2}$ and $c_{2}$ in the integrand are given in the appendix. Contributions from interference cross sections

1. $\sigma_{i}\left(\gamma^{*} q_{i} \rightarrow q_{j} \pi\right)$

The cross section for the process $\gamma^{*} q_{i} \rightarrow q_{j} \pi$ arising from the cross term is found to be of the from.

$$
\sigma_{i}\left(\gamma^{*} q_{i} \rightarrow q_{j} \pi\right)=\frac{g_{A}^{2}}{f^{2}} \frac{1}{64 \pi^{2}}\left(\frac{e_{q_{i}}}{e_{q_{j}}}\right) \frac{1}{P^{2}} \frac{1}{\left(s-m_{q}^{2}\right)} \int_{-\Lambda^{2}}^{t_{2}} d t \frac{a_{1}^{i}+b_{1}^{i} t+c_{1}^{i} t^{2}}{\left(t-m_{q}^{2}\right)}
$$

The expressions for the coefficients $a_{1}^{i}, b_{1}^{i}$ and $c_{1}^{i}$ in the integrand are given in the appendix.

2. $\gamma^{*} \pi \rightarrow q_{i} \bar{q}_{j}$

The cross section for the process $\gamma^{*} \pi \rightarrow q_{i} \bar{q}_{j}$ arising from the crossed term is found to be of the form.

$$
\sigma_{i}\left(\gamma^{*} \pi \rightarrow q_{i} \bar{q}_{j}\right)=\frac{g_{A}^{2}}{f^{2}} \frac{\sigma_{\circ}}{32 \pi^{2}}\left(1-\frac{e_{\pi}}{e_{q}}\right) \frac{1}{K^{2}} \int_{-\Lambda^{2}}^{t_{2}} d t \frac{a_{2}^{i}+b_{2}^{i} t+c_{2}^{i} t^{2}}{\left(t-m_{q}^{2}\right)\left(t+a_{\circ}^{i}\right)}
$$

where

$$
a_{\circ}^{i}=Q^{2} / z-\left(m_{q}^{2}+m_{\pi}^{2}\right)
$$


The expressions for the coefficients $a_{2}^{i}, b_{2}^{i}$ and $c_{2}^{i}$ in the integrand are given in the appendix.

The cross section for $\gamma^{*} q \rightarrow \pi q^{\prime}$ evaluated perturbatively in the lowest order leads to the evolution kernel $\mathcal{P}_{\pi q}$ which has a $z$ dependence

$$
\mathcal{P}_{\pi q}(z)=\frac{\alpha_{\pi}}{8 \pi^{2}} \sigma_{\circ} m_{q}^{2} m_{\pi}^{2} Q^{2}\left[\frac{d p^{2}}{d Q^{2}} \frac{1}{p^{4}} \int_{t_{1}}^{t_{2}} d t \frac{t}{\left(t-m_{\pi}^{2}\right)^{2}}-\frac{1}{p^{2}} \frac{t_{2}}{\left(t_{2}-m_{\pi}^{2}\right)^{2}} \frac{d t_{2}}{d Q^{2}}\right]
$$

where we have written $\alpha_{\pi}=g_{A}^{2} / f^{2}$. Other quantities are defined as follows.

$$
\begin{aligned}
& t_{2}=2 m_{q}^{2}-\sqrt{\left(P^{2}+m_{q}^{2}\right)\left(K^{2}+m_{\pi}^{2}\right)}+2 P K \\
& t_{1}=2 m_{q}^{2}-\sqrt{\left(P^{2}+m_{q}^{2}\right)\left(K^{2}+m_{\pi}^{2}\right)}-2 P K
\end{aligned}
$$

where $P$ and $K$ are the magnitudes of the three momentum of the initial hadron and pion. In the above $\Lambda_{\chi S B}$ is an ultraviolet cut off parameter [4] which may be supposed to define the scale of chiral symmetry breaking. The evolution kernel $\mathcal{P}_{q^{\prime} q}(z)$ corresponding to the process $\gamma^{*} q \rightarrow q^{\prime} \pi$ is

$$
\mathcal{P}_{q^{\prime} q}(z)=g_{d}^{q}\left(z, Q^{2}\right)+\frac{e_{q}}{e_{q}^{\prime}} f_{i}^{q}\left(z, Q^{2}\right)
$$

and the evolution kernel $\mathcal{P}_{q^{\prime} \pi}(z)$ for the process $\gamma^{*} \pi \rightarrow q^{\prime} \bar{q}$ is

$$
\mathcal{P}_{q^{\prime} \pi}(z)=g_{d}^{\pi}\left(z, Q^{2}\right)+\left(\frac{e_{\pi}}{e_{q}^{\prime}}-1\right) f_{i}^{\pi}\left(z, Q^{2}\right)
$$

The expressions for $g_{d}^{q}, g_{d}^{\pi}, f_{i}^{q}$ and $f_{i}^{\pi}$ are given in the Appendix. The quark densities are then found to satisfy the following equations

$$
\begin{aligned}
& \frac{d u\left(z, Q^{2}\right)}{d \tau}=\mathcal{P}_{u u} \otimes u+\mathcal{P}_{u d} \otimes d+\mathcal{P}_{u \pi^{\circ}} \otimes \pi^{\circ}+\mathcal{P}_{u \pi^{+}} \otimes \pi^{+} \\
& \frac{d \bar{u}\left(z, Q^{2}\right)}{d \tau}=\mathcal{P}_{\bar{u} \bar{u}} \otimes \bar{u}+\mathcal{P}_{\bar{u} \bar{d}} \otimes \bar{d}+\mathcal{P}_{\bar{u} \pi^{\circ}} \otimes \pi^{\circ}+\mathcal{P}_{\bar{u} \pi^{-}} \otimes \pi^{-}
\end{aligned}
$$

and the pion densities satisfy 


$$
\begin{gathered}
\frac{d \pi^{+}\left(z, Q^{2}\right)}{d \tau}=\mathcal{P}_{\pi^{+} u} \otimes u+\mathcal{P}_{\pi^{+} \bar{d}} \otimes \bar{d} \\
\frac{d \pi^{-}\left(z, Q^{2}\right)}{d \tau}=\mathcal{P}_{\pi^{-} d} \otimes d+\mathcal{P}_{\pi^{-} \bar{u}} \otimes \bar{u} \\
\frac{d \pi^{\circ}\left(z, Q^{2}\right)}{d \tau}=\sum \mathcal{P}_{\pi^{\circ} q_{i}} \otimes q_{i}
\end{gathered}
$$

The evolution kernels are explicitly flavor-dependent as stated in eqn(2.12). This implies that the Goldstone isotriplet has a non-vanishing contribution to the evolution of the non-singlet combinations $q=u-d$ and $\bar{q}=\bar{u}-\bar{d}$. This again is a consequence of inclusion of the interference term in the kinematic region of interest and is a feature characteristic of evolution through flavor-changing interactions. The moment equations resulting from these evolution equations will be discussed in section 5. The dependence of the evolution kernels on $Q^{2}$ is shown in Figures 1-5. The value of $\lambda_{\chi S B}$ is chosen to be $1170 \mathrm{MeV}$. 


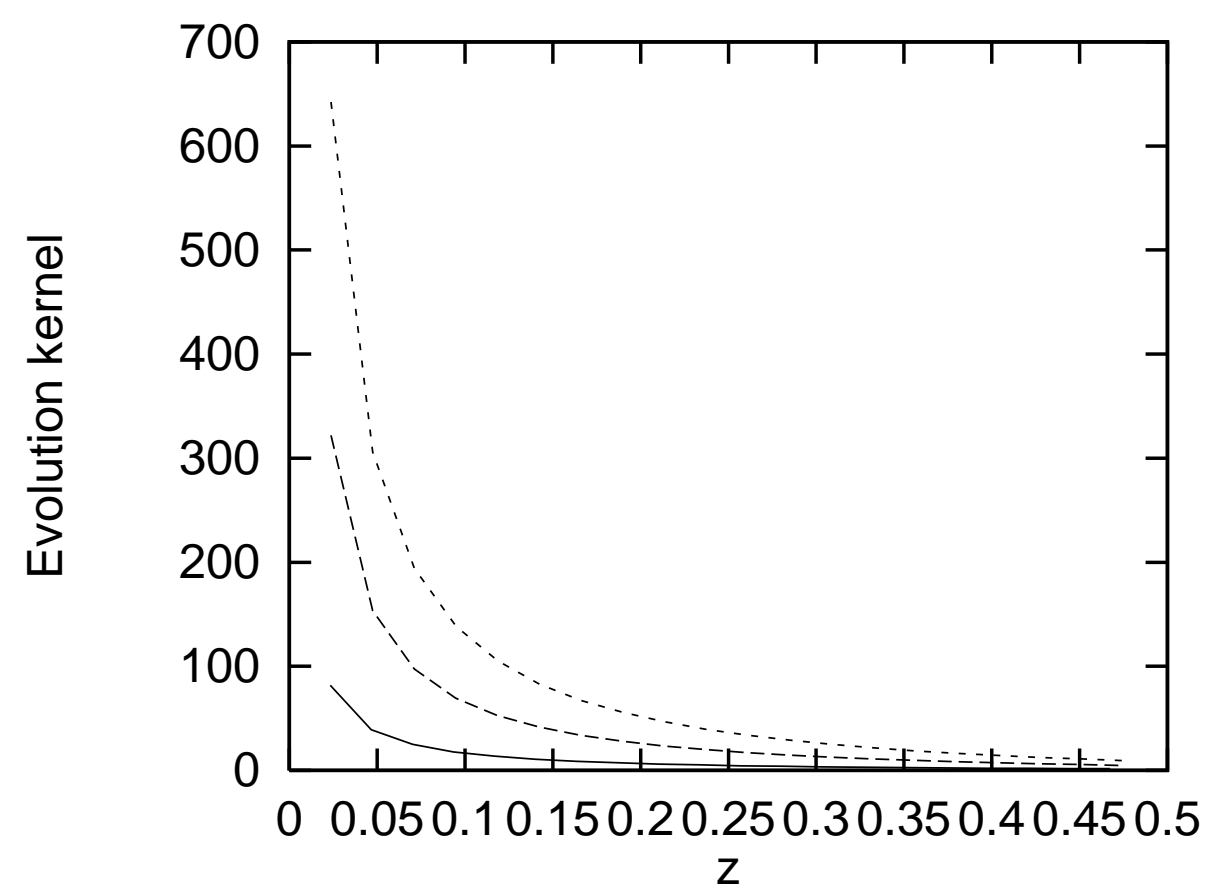

Figure 1: Contribution of the t-channel diagram to $P_{q \pi}(z)$ for $1 . Q^{2}=$ 2.0 $\mathrm{GeV}^{2}$ (solid line), 2. $Q^{2}=2.0 \mathrm{GeV}^{2}$ (dashed line), and 3. $Q^{2}=4.0 \mathrm{GeV}^{2}$ (dotted line)

\section{Probabilistic calculation of the evolution ker- nels}

In the kinematic region $x P>>Q \geq p_{T}$, where $P$ denotes the hadron momentum, the leading logarithmic approximation is known to be valid in QCD. The evolution kernels obtained in this approximation admits probabilistic interpretation and can in fact be calculated taking direct advantage of that. Durand and Putikka have shown that even the infrared divergences can be eliminated in such calculations if the 'loss terms' are correctly incorporated. In this section, we apply this method to derive the evolution equtions in the chiral field theory for the kinematic region specified above. the exercise leads to results which bring out striking differences between the evolution kernels in QCD and those in $\chi F T$. In particular, it illustrates the difficulties one would face if one tries to apply the latter to high energy.

Following Durand and Putikka, we now denote by $x$ the fraction of the hadron momentum carried by a parton. Using the vertex factor of $\chi F T$, the transition 


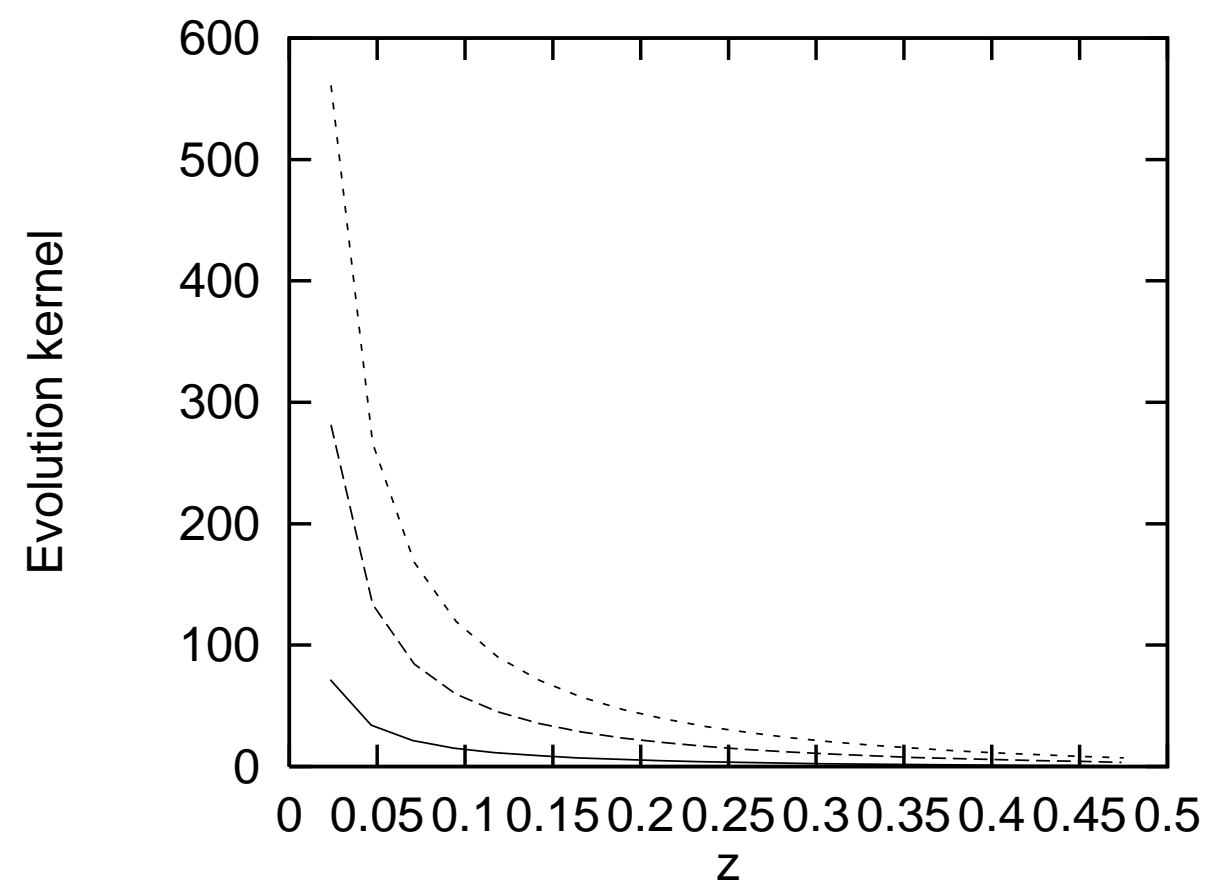

Figure 2: Contribution of the interference term to the evolution kernel $P_{q \pi}(z)$ for 1. $Q^{2}=0.5 \mathrm{GeV}^{2}$ (solid line), 2. $Q^{2}=2.0 \mathrm{GeV}^{2}$ (dashed line), and 3. $Q^{2}=$ $4.0 \mathrm{GeV}^{2}$ (dotted line). 


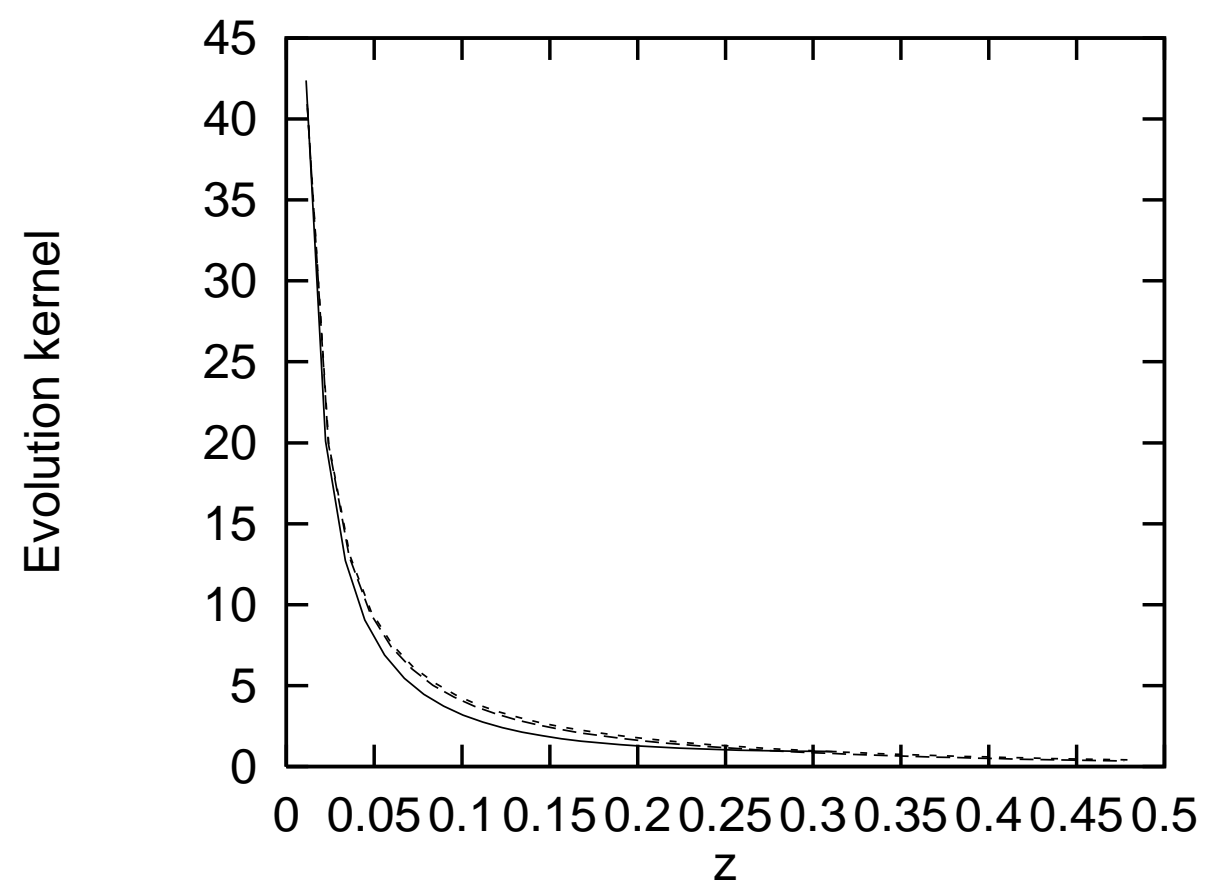

Figure 3: Contribution of the t-channel diagram to the evolution kernel $P_{j i}(z)$. The solid line corresponds to $Q^{2}=0.5 \mathrm{GeV}^{2}$ and the broken line to $Q^{2}$ between 2.0 to $4.0 \mathrm{GeV}^{2}$. 


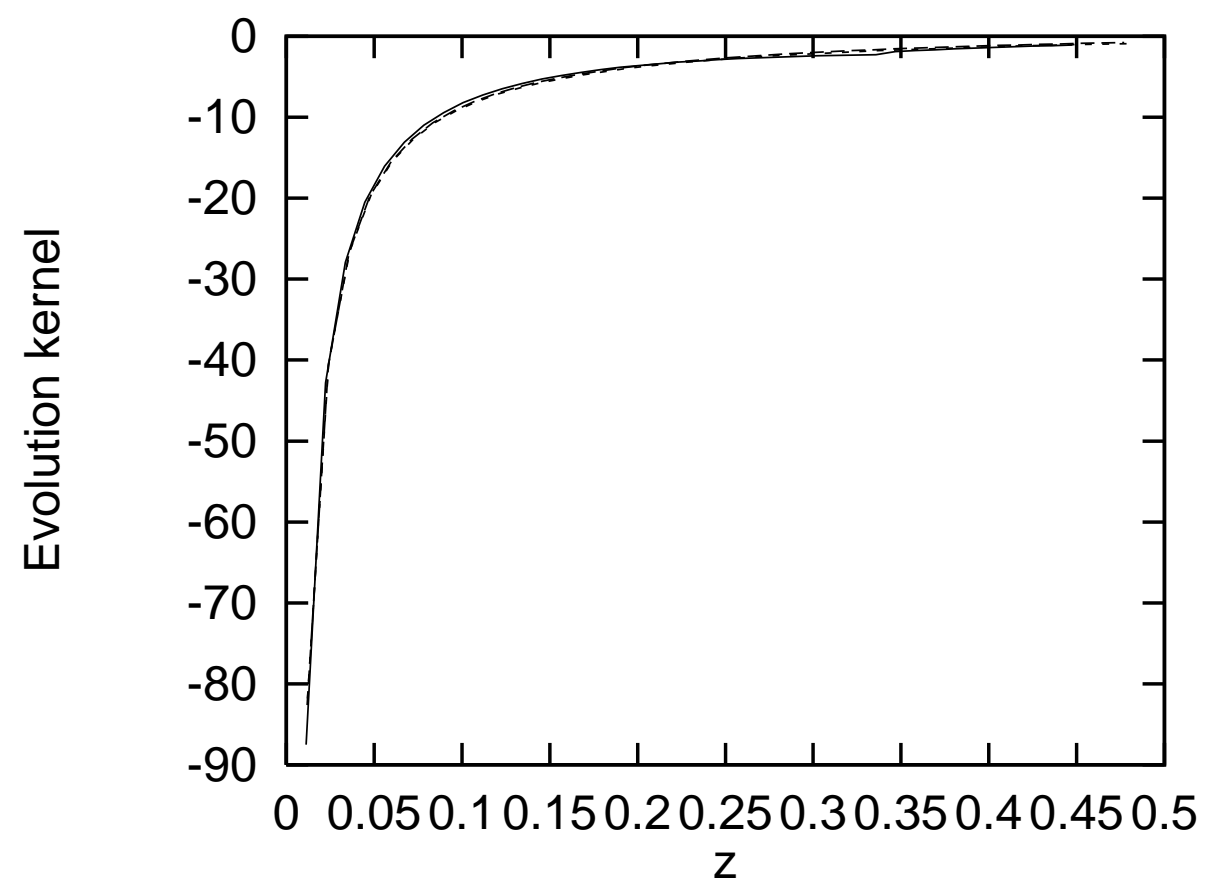

Figure 4: Contribution of the interference term to the evolution kernel $P_{i j}(z)$. The plot does not show any perceptible variation with $Q^{2}$ in the range $0.5-4.0 \mathrm{GeV}^{2}$. 


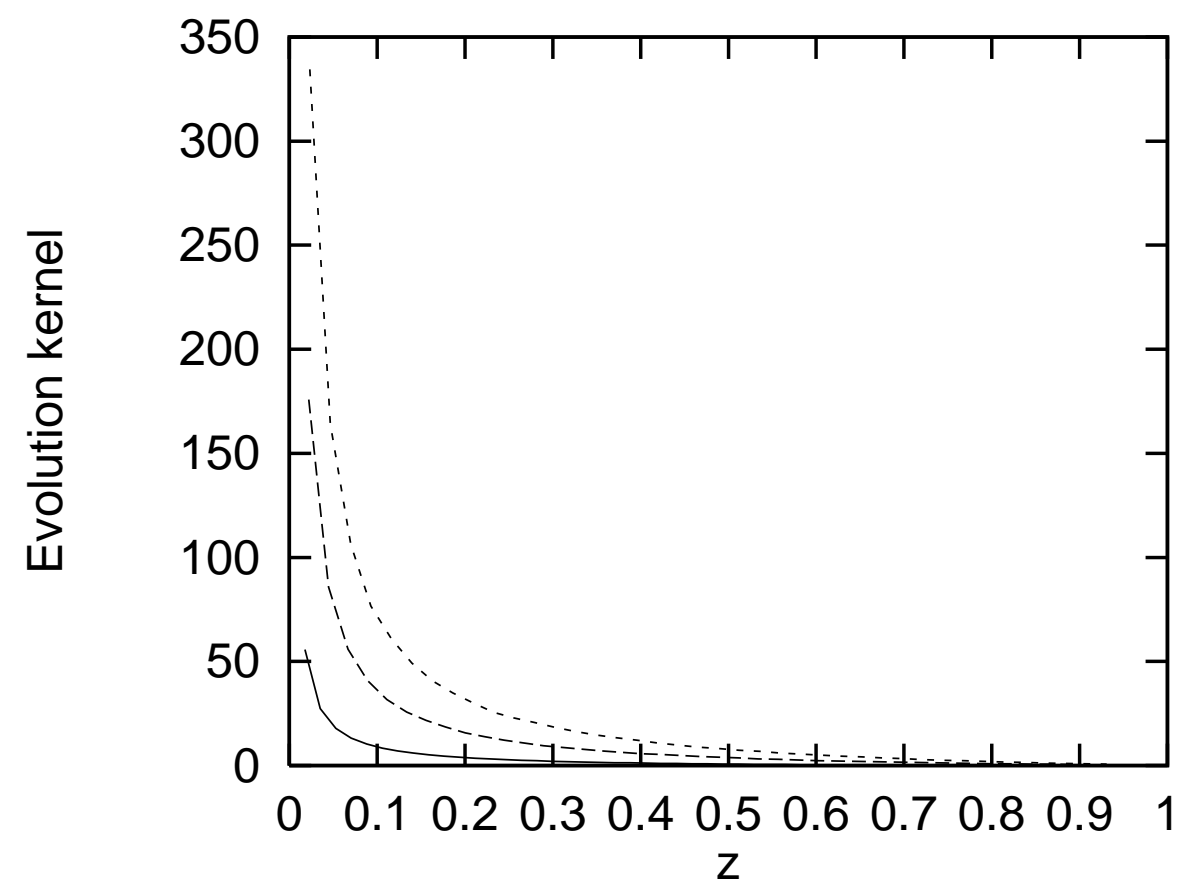

Figure 5: Variation of the evolution kernel $P_{\pi q}(z)$ with $z$ plotted for 1 . $Q^{2}=$ $0.5 \mathrm{GeV}^{2}$ (solid line), 2. $Q^{2}=2.0 \mathrm{GeV}^{2}$ (dashed line), and 3. $Q^{2}=4.0 \mathrm{GeV}^{2}$ (dotted line). 
probabilities can be calculated from these diagrams in a straightforward manner. Neglecting the quark masses and writing $\alpha_{\pi}=g_{A}^{2} / f^{2}$, the resulting evolution equations are

$$
\begin{gathered}
\frac{d u}{d \tau}=\frac{\alpha_{\pi} m_{\pi}^{2}}{(2 \pi)^{3}}\left[4 x u(x) \int_{\circ}^{x} d x_{1} \frac{x_{1}^{3}\left(x_{1}-x\right)}{\left(x_{1}-x\right)^{2}+\epsilon}\right. \\
\left.+\int_{x}^{1} d x_{1}\left[2 x_{1} x^{3} q^{+}\left(x_{1}\right)+\frac{x_{1}-x}{2 x_{1}} \pi^{(+\circ)}\left(x_{1}\right)\right] \frac{x_{1}-x}{\left(x_{1}-x\right)^{2}+\epsilon}\right] \\
\frac{d \pi^{+}}{d \tau}=\frac{\alpha_{\pi} m_{\pi}^{2}}{2 x}\left[\int_{x}^{1} \frac{d x_{1}}{x_{1}} u\left(x_{1}\right) \frac{2\left(x_{1}-x\right)^{3}}{\left(x_{1}-x\right)^{2}+\epsilon}-\pi^{+} \int_{0}^{x} d x_{1} \frac{\left(x-x_{1}\right)^{2}}{\left(x-x_{1}\right)^{2}+\epsilon}\right] \\
\frac{d \pi^{0}}{d \tau}=\frac{\alpha_{\pi} m_{\pi}^{2}}{x}\left[\int_{x}^{1} \frac{d x_{1}}{x} q^{+}\left(x_{1}\right) \frac{\left(x_{1}-x\right)^{3}}{\left(x_{1}-x\right)^{2}+\epsilon}-\pi^{0} \int_{0}^{x} d x_{1} \frac{\left(x-x_{1}\right)^{2}}{\left(x-x_{1}\right)^{2}+\epsilon}\right]
\end{gathered}
$$

where $\epsilon=p_{T}^{2} / 2 P^{2}, q^{+}=u+d$ and $\pi^{(+\circ)}=\pi^{+}+\pi^{\circ}$.

There are obvious differences between these equations and those obtained in QCD by the same procedure. The most important difference can be seen if one considers the limit $\epsilon \rightarrow 0$. Whereas inclusion of loss terms leads to elimination of infra-red divergences in the splitting functions in the case of QCD evolution, the divergence in the $\epsilon \rightarrow 0$ limit is not eliminated in the $\chi \mathrm{FT}$ evolution. This shows that the kinematic region corresponding to this limit is beyond the domain of applicability of the chiral field theory which is not valid above the chiral symmetry breaking scale. This is true for all such theories which would incorporate evolution through emission of chiral Goldstone bosons.

\section{Evolution of moments}

The $Q^{2}$-evolution of the moments of the structure functions in the chiral field theory follows directly from the basic equations (36) - (40). However the resulting evolution is not as simple as in the case of the leading logarithmic approximation. Complication arises due to two distinctive features of the basic evolution equations. Firstly, the kernels of evolution are $Q^{2}$-dependent. Secondly, the flavor-dependence of the pionic contribution makes it harder to decouple the moment equations. In particular, simple non-singlet combinations like $q-\bar{q}$ no longer evolve in purely multiplicative manner.

We introduce the moments of the parton densities and the evolution kernel by

$$
Q_{n}(\tau)=\int_{0}^{1} d x x^{n-1} q_{n}(x, \tau)
$$




$$
\gamma_{n}{ }^{A B}(\tau)=\int_{0}^{1} d x x^{n-1} \mathcal{P}_{A B}(x, \tau)
$$

and take moments of the equations (36) - (40) to obtain

$$
\begin{aligned}
\frac{d}{d \tau} U_{n}(\tau) & =\gamma_{n}{ }^{u u} U_{n}+\gamma_{n}{ }^{u d} D_{n}+\gamma_{n}{ }^{u \pi^{\circ}} \Pi_{n}^{\circ}+\gamma_{n}{ }^{u \pi^{+}} \Pi_{n}{ }^{+} \\
\frac{d}{d \tau} \bar{U}_{n}(\tau) & =\gamma_{n}{ }^{\bar{u} \bar{u}} \bar{U}_{n}+\gamma_{n}{ }^{\bar{u} \bar{d}} \bar{D}_{n}+\gamma_{n}{ }^{\bar{u} \pi^{\circ}} \Pi_{n}^{\circ}+\gamma_{n}{ }^{\bar{u} \pi^{-}} \Pi_{n}{ }^{-} \\
\frac{d}{d \tau} \Pi_{n}{ }^{+}(\tau) & =\gamma_{n}{ }^{\pi^{+} u} U_{n}+\gamma_{n}{ }^{\pi^{+} \bar{d}} \bar{D}_{n} \\
\frac{d}{d \tau} \Pi_{n}{ }^{\circ}(\tau) & =\sum \gamma_{n}{ }^{\pi^{\circ}} q_{i} Q_{i n}
\end{aligned}
$$

and similar equations for $D_{n}, \bar{D}_{n}$, and $\Pi_{n}{ }^{-}$.

We shall consider below the exact solution to these equations first and then make an approximation to bring out clearly the role of the pion decomposition process in the evolution of the moments. We shall omit the index $n$ from the symbols $Q_{n}, \gamma_{n}^{q q}$ etc. in the expressions given below for reasons of typographical simplicity.

\subsection{Exact solutions}

Define the combinations $U^{(-)}=U-\bar{U}, \Pi^{(-)}=\Pi^{+}-\Pi^{-}$etc. and transform to

$$
u^{(-)}=\exp \left(\int_{\tau_{0}}^{\tau} d \tau \gamma^{u u}\right) U^{(-)}
$$

Further writing

$$
X=\left(\begin{array}{c}
u^{(-)} \\
d^{(-)} \\
\Pi^{(-)}
\end{array}\right)
$$

a formal solution is obtained as

$$
X(\tau)=\exp \left(\int_{\tau_{0}}^{\tau} M d \tau\right) X\left(\tau_{0}\right)
$$

where

$$
M=\left(\begin{array}{ccc}
0 & \tilde{\gamma}^{u d} & \gamma^{u \pi} \\
\tilde{\gamma}^{d u} & 0 & \tilde{\gamma}^{d \pi} \\
\tilde{\gamma}^{\pi u} & \tilde{\gamma}^{\pi d} & 0
\end{array}\right)
$$

The evolution takes a simple form for the eigenfunctions of the matrix $M$. Therefore the first task is to diagonalize it. After diagonalization one finds that there are three eigenfunctions of evolution given by

$$
v_{i}(\tau)=u^{(-)}+\eta d^{(-)}+\zeta \Pi^{(-)}
$$


where

$$
\begin{gathered}
\eta=\frac{\lambda_{i} \Gamma^{d u}+\Gamma^{d \pi} \Gamma^{\pi u}}{\lambda_{i}^{2}+\tilde{\Gamma}^{d \pi} \Gamma^{\pi d}} \\
\zeta=\frac{1}{\lambda_{i}}\left[\Gamma^{\pi u}-\Gamma^{\pi d} \frac{\lambda_{i} \Gamma^{d u}+\Gamma^{d u} \Gamma^{\pi u}}{\lambda_{i}^{2}+\Gamma^{d \pi} \Gamma^{\pi d}}\right] \\
\Gamma^{i j}=\int_{\tau_{1}}^{\tau} d \tau \gamma^{i j}
\end{gathered}
$$

The $\lambda_{i}$ 's, being the three eigenvalues of $M$, are the roots of the cubic equation

$$
\begin{gathered}
\lambda^{3}-\alpha \lambda+\beta=0 \\
\alpha=\tilde{\gamma}^{u \pi} \gamma^{\pi u}-\tilde{\gamma}^{d \pi} \gamma^{\pi d}-\tilde{\gamma}^{d u} \tilde{\gamma}^{u d} \\
\beta=\tilde{\gamma}^{d u} \tilde{\gamma}^{u \pi} \gamma^{\pi u}-\tilde{\gamma}^{u d} \gamma^{d \pi} \gamma^{\pi u}
\end{gathered}
$$

The evolution of each such combination $v_{i}(\tau)$ is then given by

$$
v_{i}(\tau)=v_{i}\left(\tau_{1}\right) \exp \left(\lambda_{i}\left(\tau, \tau_{1}\right)\right.
$$

It may be noted that the quantities $\Gamma^{i j}$ are simply related to the moments of the cross section. Thus, putting back the moment index,

$$
\Gamma_{n}^{i j}=\sigma_{n}^{i j}(\tau)-\sigma_{n}^{i j}\left(\tau_{1}\right)
$$

\subsection{The role of the pion splitting function}

Influnce of the pion splitting kernel on the evolution of the non-singlet first moment can be clearly shown in a simplified approximate version of the moment equations(5.81)-(5.84). Let us introduce the symbols $\mathcal{P}_{q q}^{D}=\mathcal{P}_{q_{i} q_{j}}, \mathcal{P}_{q q}^{N D}=\mathcal{P}_{q_{i} q_{j}}(i \neq$ $j), \mathcal{P}_{q \bar{q}}^{D}=\mathcal{P}_{q_{i} \bar{q}_{i}}^{D}, Q_{q q}=\mathcal{P}_{q q}^{D}-\mathcal{P}_{q q}^{N D}, Q_{q \bar{q}}=\mathcal{P}_{q \bar{q}}^{D}-\mathcal{P}_{q \bar{q}}^{N D}$ where we assume the validity of the isospin $\mathrm{SU}(2)$ symmetry and charge conjugation invariance. The moments of the kernels defined above will be represented by $\gamma_{n}^{D}$ etc, in obvious analogy with(5.80). Further, if we take the combination

$$
q^{+}=(u-d)+(\bar{u}-\bar{d})
$$


and neglect the flavour-dependence of all other kernels except that of $\mathcal{P}_{q \pi}$, the evolution equation for the combination $q^{+}$of the quark and antiquark densities takes the simple form.

$$
\frac{d}{d \tau} q^{+}\left(z, Q^{2}\right)=Q_{q q} \otimes q^{+}+\mathcal{P}_{\pi}^{\prime} \otimes\left(\pi^{+}-\pi^{-}\right)
$$

where $\mathcal{P}_{\pi}^{\prime}=\mathcal{P}_{u \pi^{+}}-\mathcal{P}_{d \pi^{+}}$. Now $Q_{q \bar{q}}$ may be neglected as it involves a process which is second order in $\alpha_{\pi}$. The equation for the first moment is then given by

$$
\frac{d}{d \tau} Q_{1}^{+}=\left(\gamma_{q q}^{D}-\gamma_{q q}^{N D}\right) Q_{1}^{+}+\gamma_{\pi}^{\prime} \Pi_{1}
$$

This equation can be solved to yield

$$
Q_{1}^{+}(\tau)=\Delta\left(\tau-\tau_{\circ}\right)\left[Q_{1}^{+}\left(\tau_{\circ}\right)+\int_{\tau_{\circ}}^{\tau} d \tau^{\prime} \gamma_{\pi}^{\prime}\left(\tau^{\prime}\right) \Pi_{1}\left(\tau^{\prime}\right) \Delta\left(\tau^{\prime}, \tau_{\circ}\right)\right]
$$

where the evolution factor is given by

$$
\Delta\left(\tau, \tau_{\circ}\right)=\exp \int_{\tau_{\circ}}^{\tau}\left[\gamma_{q q}^{D}\left(\tau^{\prime}\right)-\gamma_{q q}^{N D}\left(\tau^{\prime}\right)\right] d \tau^{\prime}
$$

This also turns out to be

$$
\begin{gathered}
\Delta\left(\tau, \tau_{\circ}\right)=A \exp \left[\frac{1}{2}{\hat{\sigma_{1}}}^{\pi^{\circ}}(\tau)-{\hat{\sigma_{1}}}^{\pi^{+}}(\tau)\right] \\
A=\exp \left[\hat{\sigma}_{1}^{\pi^{+}}\left(\tau_{\circ}\right)-\frac{1}{2}{\hat{\sigma_{1}}}^{\pi^{\circ}}\left(\tau_{\circ}\right)\right]
\end{gathered}
$$

In the low $Q^{2}$ region, the pion density may be supposed to be small enough for one to neglect the second term in (55) in comparison with the first term. This situation corresponds to a multiplicative evolution of the Gottfried sum which evolves from the quark model value to a lower value at moderate $Q^{2}$, as observed experimentally[2]. At higher $Q^{2}$, the evolution becomes more complicated due to the presence of the second term.

\section{Conclusion}

One can obtain $Q^{2}$-evolution of the moments of the structure functions in the chiral field theory at low $Q^{2}$, and the Gottfried sum does show variation with $Q^{2}$. However, the kinematic region in which the theory is applicable makes it difficult to compare the results of the theory with the NMC and the other experimental results. Further, unlike the splitting functions in the Altarelli-Parisi formalisim, the evolution kernels in such effective theories admit simple probabilistic interpretation. 


\section{Appendix}

Given below are the coefficients appearing in the different cross sections in section 3. Coefficients of the cross section for the process $\gamma^{*} q_{i} \rightarrow q_{j} \pi$

$$
\begin{aligned}
& a_{1}=m_{q}^{4}\left(s+Q^{2}\right)-4 m_{q}^{2} m_{\pi}^{2} Q^{2}-m_{q}^{4}\left(4 m_{q}^{2}-3 m_{\pi}^{2}\right) \\
& b_{1}=-2 m_{q}^{2}\left(s+Q^{2}\right)+3 m_{q}^{2}\left(3 m_{q}^{2}+2 m_{\pi}^{2}\right) \\
& c_{1}=s+Q^{2}-6 m_{q}^{2}-m_{\pi}^{2} \\
& d_{1}=1
\end{aligned}
$$

Coefficients of the cross section for the process $\gamma^{*} \pi \rightarrow q_{i} \bar{q}_{j}$

$$
\begin{aligned}
& a_{2}=m_{q}^{4}\left(s+2 m_{q}^{2}\right)+4 m_{q}^{2} m_{\pi}^{2}\left(Q^{2}-m_{q}^{2}\right) \\
& b_{2}=-2 m_{q}^{2}\left(s+2 m_{q}^{2}\right)-4 m_{q}^{2} m_{\pi}^{2} \\
& c_{2}=s+2 m_{q}^{2}
\end{aligned}
$$

Coefficients of the cross section for the crossed channel process $\gamma^{*} q_{i} \rightarrow q_{j} \pi$

$$
\begin{aligned}
a_{1}^{i}= & m_{q}^{2}\left(Q^{2}+2 m_{q}^{2}+3 m_{\pi}^{2}\right)\left(s-m_{q}^{2}-m_{\pi}^{2}\right)-m_{q}^{2}\left(m_{q}^{2}-m_{\pi}^{2}\right)\left(s+3 m_{q}^{2}-m_{\pi}^{2}\right) \\
& +m_{q}^{2}\left(Q^{2}+m_{q}^{2}\right)\left(s+m_{\pi}^{2}-m_{q}^{2}\right)-2 m_{q}^{2}\left(s\left(s-2 m_{q}^{2}-m_{\pi}^{2}\right)+m_{q}^{2}\left(m_{q}^{2}-m_{\pi}^{2}\right)\right) \\
& -4 m_{q}^{2} m_{\pi}^{2}\left(s-2 m_{q}^{2}+m_{\pi}^{2}\right) \\
b_{1}^{i}= & -2\left(s-m_{q}^{2}\right)\left(s+Q^{2}-m_{q}^{2}-m_{\pi}^{2}\right)+4 m_{q}^{2}\left(2 m_{q}^{2}+m_{\pi}^{2}\right) \\
c_{1}^{i}= & -2\left(s+m_{q}^{2}\right)
\end{aligned}
$$

Coefficients of the cross section for the crossed channel process $\gamma^{*} \pi \rightarrow q_{i} \bar{q}_{j}$

$$
\begin{aligned}
a_{2}^{i}= & -m_{q}^{2}\left(Q^{2}+m_{q}^{2}\right)\left(s-2 m_{q}^{2}\right)-m_{q}^{2}\left(Q^{2}+m_{q}^{2}\right)\left(s+2 Q^{2}-2 m_{\pi}^{2}\right)-2 m_{q}^{2}\left(s+Q^{2}-m_{q}^{2}\right)^{2} \\
& +2 m_{q}^{2} m_{\pi}^{2}\left(s+Q^{2}+m_{q}^{2}\right)-4 m_{q}^{2} m_{\pi}^{2}\left(2 m_{\pi}^{2}+3 m_{q}^{2}\right)+8 m_{q}^{2} m_{\pi}^{2}\left(s+Q^{2}\right) \\
b_{2}^{i}= & -\left(s-2 m_{q}^{2}\right)\left(2 s+Q^{2}-2 m_{q}^{2}-2 m_{\pi}^{2}\right)-s\left(Q^{2}+m_{q}^{2}\right)-m_{q}^{2}\left(s+2 Q^{2}-2 m_{\pi}^{2}\right) \\
& -2 m_{q}^{2}\left(2 s+2 Q^{2}-2 m_{\pi}^{2}-m_{\pi}^{2}\right)+8 m_{q}^{2} m_{\pi}^{2} \\
c_{2}^{i}= & -2 s
\end{aligned}
$$




\section{References}

1. G.Altarelli, Phys. Rep.,81 (1982).

2. P.Amaudruz et al., Phys. Rev. Lett. 66 (1991) 2712;

M.Arnedo et al., Phy.Rev. D 50(1994)R1;

M.R.Adams et al.(E 665 collaboration), Phys.Rev.Lett. 75(1995)1466;

Phys.Rev. D 54(1996)3006;

K.Ackerstaff(hermes collaboration),DESY- HERMES-96-01(1996)

3. A.Baldit et al.(NA51 collaboration), Phys.Lett. B332(1994)244.

4. For a comprehensive review, see S.Kumano, Phys.Rep. 303(1998),183.

5. A.W.Thomas, Nucl. Phys. A532 (1991) 271;

A.Signal, A.W.Schreiber and A.W.Thomas, Mod. Phys. Lett. A6 (1991) 271.

6. E.J.Eichten, I. Hinchliffe and C. Quigg, Phys. Rev. D45 (1992) 2269.

7. E.M.Henley and G.A.Miller, Phys.Lett. B251(1990)453;

S.Kumano, Phys.Rev. D43(1991)59;

S.Kumano and J.T.Londergan, Phys. Rev. D44 (1991) 271.

8. R.D.Ball and S.Forte, Nucl. Phys. B425 (1994) 516.

9. K.Roy-Maity and P.Dasgupta, Int. J. Mod. Phys. A13 (1998) 1785.

10. H.Georgi and A.V.Manohar, Nucl. Phys. B234 (1984) 189. 